\title{
Características sócio-demográficas, condições de saúde e utilização de serviços de saúde por idosos ${ }^{1}$
}

\author{
Features socio-demographic, conditions of health and the use of the \\ health system for elderly
}

\section{Características sociodemográficas, condiciones de salud y utilización de los servicios de salud para ancianos}

\author{
Darlene Mara dos Santos Tavares ${ }^{\mathrm{I}}$, Gustavo Emanuel Carvalho Borela Guidetti ${ }^{\mathrm{II}}$, \\ Maria Isabel Borges Moreira Saúde ${ }^{\mathrm{III}}$
}

\section{RESUMO}

Essa pesquisa objetivou identificar as características sócio-demográficas e de saúde, bem como utilização de serviços de saúde por idosos residentes na zona urbana de um município do interior de Minas Gerais, e comparar estas variáveis entre os três distritos sanitários. Inquérito domiciliar, transversal e observacional, realizado entre 2005 e 2006 em um município do interior de Minas Gerais. Participaram 2.924 idosos, entrevistados no domicílio, selecionados através da técnica de amostragem estratificada. Os dados foram digitados no EpiInfo 3.2., realizando-se análise descritiva, testes $X 2$ e exato de Fisher $(p<0,05)$. O percentual de idosos nos três distritos sanitários foi: $33,5 \%$ para o DSI; $35,7 \%$ para o DSII e $30,8 \%$ para o DSIII. Maioria sexo feminino (64\%); faixa etária de $60 \mid-70$ anos $(52,7 \%)$; moram com esposo ou companheiros $(47 \%)$. Os idosos residentes no DSI quando comparados aos do DSII e DSIII apresentam maiores desafios na atenção à sua saúde, no entanto, estão mais satisfeitos com o atendimento e internações.

Palavras chave: Idoso; Serviço de saúde; Planejamento em saúde; Serviços de saúde para idosos; Necessidades e demandas de serviços de saúde; Diagnóstico da situação em saúde.

\section{ABSTRACT}

This research objectives identify the sociodemographic characteristics and health and use of the service health of elderly residents in the village of the municipality in the interior of Minas Gerais and compare these variables between the three districts health. Inquiry home, transverse and observational, conducted between 2005 and 2006 in a municipality in the interior of Minas Gerais. Participating 2,924 elderly, interviewed at home, selected by stratified sampling technique. The data were entered in EpiInfo 3.2. By conducting themselves descriptive analysis, tests $x 2$ and Fisher's exact $(p<0.05)$. The percentage of elderly people in the three districts health was: $33.5 \%$ for the DSI; $35.7 \%$ for the DSII and $30.8 \%$ for the DSIII. Majority females (64\%); age of $60 \mid-70$ years $(52.7 \%)$ live with spouse or companions $(47 \%)$. The elderly residents in the DSI when compared to the DSII and DSIII present major challenges in attention to their health, however, are more satisfied with the care and hospitalization.

Key words: Aged; Health services; Health planning; Health services for the aged; Health services needs and demand; Diagnosis of health situation.

\section{RESUMEN}

Este estudio tuvo como objetivo identificar las características socio-demográficas y de salud, y la utilización de los servicios de salud para los ancianos residentes en la zona urbana de un municipio en el interior de Minas Gerais, y comparar estas variables entre los tres distritos sanitarios. Investigación domiciliaria, transversal y observacional, realizado entre 2005 y 2006 en un municipio en el interior de Minas Gerais. Participaron 2924 ancianos, entrevistados en su casa, seleccionados

\footnotetext{
Esta pesquisa recebeu apoio financeiro da Fundação de Amparo à Pesquisa do Estado de Minas Gerais (FAPEMIG)

${ }^{I}$ Enfermeira. Mestre e Doutor em Enfermagem. Professora Adjunto do Departamento de Enfermagem em Educação e Saúde Comunitária do Curso de Graduação em Enfermagem da Universidade Federal do Triângulo Mineiro (UFTM). Email: darlenetavares@netsite.com.br

II Enfermeiro. Graduado pelo Curso de Graduação em Enfermagem. Universidade Federal do Triângulo Mineiro.

III Médica. Mestre e Doutora em Saúde Pública. Médica do Departamento de Medicina Social. Curso de Medicina. Universidade Federal do Triângulo Mineiro.
} 
Tavares DMS, Guidetti GECB, Saúde MIBM. Características sócio-demográficas, condições de saúde e utilização de serviços de saúde por idosos. Revista Eletrônica de Enfermagem [Internet]. 2008;10(2):299-309. Available from: http://www.fen.ufg.br/revista/v10/n2/v10n2a02.htm

mediante técnica de muestreo estratificado. Los datos fueron introducidos en EpiInfo 3,2.,y fueron analizados a través de teste quicuadrado y exacta de Fisher $(p<0,05)$. El porcentaje de ancianos en los tres distritos de salud fue: $33,5 \%$ para la DSI, 35,7\% para DSII y $30,8 \%$ para el DSIII. Mayoría mujeres (64\%); edad de 60 años | - 70 años (52,7\%); vivir con su cónyuge o compañero (47\%). Los ancianos residentes en la DSI si se compara con la DSII y

\section{NTRODUÇÃO}

O envelhecimento da população é um dos maiores triunfos da humanidade e também um dos grandes desafios. No século XXI, o envelhecimento global causará aumento nas demandas sociais e econômicas em todo o mundo $^{(1)}$. A redução das taxas de mortalidade e fecundidade, nas últimas décadas do século passado, mudou o perfil demográfico do Brasil. Rapidamente, deixou-se de ser um "país de jovens" e o envelhecimento tornou-se questão fundamental para as políticas públicas. Os brasileiros com mais de 60 anos representam $10,2 \%$ da população, devendo chegar a $14 \%$ em 2025. O envelhecimento populacional muda o perfil de adoecimento e traz repercussões para a atenção à saúde e para as políticas públicas, que passam a ter que enfatizar a promoção da saúde, a manutenção da autonomia e a valorização das redes de suporte social $^{(2)}$.

O envelhecimento populacional ocorre de forma desigual no Brasil. Aproximadamente $45 \%$ dos idosos residem nos Estados de São Paulo (23\%), Rio de Janeiro (11,5\%) e Minas Gerais $(11,2 \%)^{(3)}$. O município de Uberaba, situado no Estado de Minas Gerais, apresenta população idosa de $10,3 \%{ }^{(4)}$. Tais dados reforçam a necessidade de se desenvolverem pesquisas neste município procurando compreender a dinâmica do seu envelhecimento populacional para a reorganização da atenção aos idosos ${ }^{(5)}$.

Em consonância com este quadro que se delineia para a população idosa no Brasil, o Ministério da Saúde aprovou a Política Nacional de Saúde da Pessoa Idosa ${ }^{(6)}$, cuja finalidade é recuperar, manter e promover a autonomia e a independência dos idosos, através do
DSIII presentes los principales retos en la atención a su salud, sin embargo, están más satisfechos con la atención y las hospitalizaciones.

Palabras clave: Anciano; Servicios de salud; Planificación en salud; Servicios de salud para ancianos; Necesidades y demandas de servicios de salud; Diagnóstico de la situación en salud.

desenvolvimento de ações de saúde individuais e coletivas, em consonância com os princípios e diretrizes do Sistema Único de Saúde (SUS) ${ }^{(7)}$. As ações em saúde do idoso devem objetivar ao máximo a manutenção do idoso na comunidade, junto de sua família, da forma mais digna e confortável possível ${ }^{(8)}$. A incapacidade funcional tem se tornado um desafio a ser enfrentado, uma vez que a expectativa de vida tem aumentado levando ao conseqüente crescimento de idosos com incapacidade funcional, diminuição do relacionamento com amigos e das atividades de lazer, que contribuem para o desenvolvimento pessoal e coletivo $^{(5)}$.

Envelhecer com qualidade é um privilégio que envolve mudanças em vários setores da sociedade e, de modo abrangente, em concepções generalizadas sobre saúde, educação, justiça e direitos sociais. O trabalho conjunto de todas as instituições envolvidas carece de determinação política e de melhor discussão das competências necessárias aos diversos profissionais nas várias instâncias. Muitas são as políticas que focalizam o idoso e sua família; porém, as dificuldades na implementação abrangem desde a precária captação de recursos ao frágil sistema de informação para a análise de condições de vida e de saúde, passando, evidentemente, pela inadequada capacitação de recursos humanos ${ }^{(7)}$.

Os constantes desafios sociais, financeiros e políticos impostos aos municípios, têm exigido dos seus gestores uma atuação mais competente para propiciar qualidade de vida adequada à comunidade. A participação popular na gestão municipal com os valores positivos dos cidadãos, a competência profissional dos servidores públicos, os planejamentos 
Tavares DMS, Guidetti GECB, Saúde MIBM. Características sócio-demográficas, condições de saúde e utilização de serviços de saúde por idosos. Revista Eletrônica de Enfermagem [Internet]. 2008;10(2):299-309. Available from: http://www.fen.ufg.br/revista/v10/n2/v10n2a02.htm

municipais e as informações sistematizadas podem facilitar a gestão das prefeituras. Esses fatores integrados podem diminuir a exclusão social nos municípios e aumentar participação política dos cidadãos, visando a sua adequada qualidade de $\operatorname{vida}^{(9)}$.

Para que o município possa realizar o planejamento das ações em saúde, de forma ascendente, pautado nas reais necessidades de saúde de seus cidadãos, devem-se somar o controle social, a disponibilidade política, a competência técnica e disponibilidade das informações em saúde - fidedignas e baseadas em dados primários e secundários.

Assim, investigações sobre a utilização do Sistema Público de Saúde podem desvelar situações que contribuirão na formulação de ações e políticas públicas, fortalecendo os Conselhos Gestores e melhorando as condições de saúde e de vida da população(10).

O município de Uberaba-MG é referência na área da saúde para 27 municípios que compõem a Gerência Regional de Saúde do Triângulo Sul. Dados da Prefeitura Municipal informam que, em 2006, 19,92\% da receita total do município $(R \$ 36.829 .011,00)$ foi investida em saúde, estando acima do mínimo preconizado pela Emenda Constitucional n029 $(15 \%)$. Os indicadores apontam que a taxa de mortalidade infantil caiu para 14,15 para 1.000 nascidos vivos, e houve aumento da expectativa de vida para 73 anos $^{(11)}$.

O sistema de saúde do município é composto por serviços próprios, contratados ou conveniados. A atenção primária do município conta com 28 unidades básicas de saúde urbanas, das quais 12 estão estruturadas sob a Estratégia da Saúde da Família distribuídas em três Distritos Sanitários; sete unidades básicas de saúde rural; 41 equipes de saúde da família. A atenção secundária possui dois ambulatórios de pronto atendimento e 11 de especialidades; Centros de acompanhamento, apoio e reabilitação, entre os quais destacam-se o Centro de Fisioterapia e Neurologia, Centro de Reabilitação da Universidade Federal do Triângulo Mineiro (UFTM), Associação de Pais e Amigos de Excepcionais (APAE), Fundação Caminhar, Associação das Crianças Deficientes $(A C D)$, Associação dos Deficientes Físicos de
Uberaba (ADEFU), Associação Mineira de Equoterapia (AME), entre outros. $A$ atenção terciária possui 11 hospitais conveniados ao Sistema Único de Saúde. Têm-se ainda três serviços de laboratório de análises clínicas contratados $^{(11)}$.

Tendo em vista a Política Nacional do Idoso e o avanço do envelhecimento populacional, justifica-se a necessidade de desenvolver pesquisas sobre a temática. Considerando ainda os percentuais do município de Uberaba-MG, o crescente consumo de serviços de saúde pelos idosos, a rede de atenção à saúde existente no município e a necessidade premente de reorganizar a atenção à saúde desta população, visando atender suas especificidades, essa pesquisa tem como objetivo: descrever as características sócio-demográficas, condições de saúde e utilização de serviços de saúde por idosos residentes no município de Uberaba; comparar estas variáveis entre os três distritos sanitários.

\section{PROCEDI MENTOS METODOLÓGI COS}

Este estudo é parte de um estudo maior, do tipo inquérito domiciliar, transversal e observacional, realizado na zona urbana do município de Uberaba-MG, que está dividido em três distritos sanitários, que por sua vez subdividem-se em dezoito áreas de abrangências.

$\mathrm{Na}$ época da coleta dos dados (2005 e 2006), a população total de idosos residentes na zona urbana era de 24.714 habitantes, estimada pela Secretaria Municipal de Saúde, baseada em dados do IBGE ${ }^{(12)}$. Os critérios de inclusão adotados foram: ter 60 anos ou mais de idade; sexo masculino ou feminino; morar na zona urbana do município e concordar em participar da pesquisa.

A amostra populacional foi calculada em 2.892 idosos considerando $95 \%$ de confiança, $80 \%$ de poder do teste, margem de erro de $4,0 \%$ para as estimativas intervalares e uma proporção estimada de $\Pi=0,5$ para as proporções de interesse.

Para o cálculo dos idosos que fariam parte da amostra em cada bairro, utilizou-se a técnica de amostragem estratificada proporcional. Naqueles bairros aonde o cálculo amostral foi 
Tavares DMS, Guidetti GECB, Saúde MIBM. Características sócio-demográficas, condições de saúde e utilização de serviços de saúde por idosos. Revista Eletrônica de Enfermagem [Internet]. 2008;10(2):299-309. Available from: http://www.fen.ufg.br/revista/v10/n2/v10n2a02.htm

inferior a cinco, considerou-se, pelo menos, dez idosos e utilizou-se a técnica de amostragem sistemática para selecionar os domicílios nos quais os idosos foram entrevistados. Portanto, considerou-se uma amostra inicial de 3.034 idosos e, com as perdas, obteve-se uma amostra final de 2.924 idosos.

Em cada bairro, foi calculado o Intervalo Amostral (IA) e, por meio de sorteio aleatório, foi selecionado o primeiro domicílio a ser visitado na primeira quadra do bairro. No sentido horário os demais domicílios foram considerados em cada quadra até percorrer o bairro todo. Nos domicílios em que não havia idoso, considerou-se o domicílio imediatamente posterior. Realizou-se um sorteio aleatório quando, na residência selecionada, havia mais de um idoso. Quando no domicílio selecionado havia idoso, mas este não estava em casa, agendou-se outro horário e, se no dia agendado o idoso não estivesse, considerou-se o domicílio imediatamente posterior. Em condomínios (prédios ou casas), o entrevistador apresentouse na portaria e questionou sobre o número de apartamentos ou casas existentes, elaborou um roteiro e deu-se seqüência na seleção dos domicílios. Utilizou-se o mapa de cada bairro disponibilizado pelo Centro de Controle de Zoonoses do Município de Uberaba, como itinerário para a seleção dos domicílios. Quando todo o bairro foi percorrido e não se alcançou o número de idosos desejado, voltou-se ao início do bairro na casa subseqüente à primeira casa selecionada, e repetiram-se todos os procedimentos até se obter o número desejado de idosos.

Para a coleta dos dados, foram selecionados dez entrevistadores, com experiência prévia, os quais foram treinados para a realização das entrevistas, incluindo o preenchimento dos instrumentos e dos relatórios, assim como a abordagem ao entrevistado. Reuniões sistemáticas ocorreram para supervisão e orientações necessárias. Foi elaborado manual de orientação para a realização das entrevistas.

Os dados foram coletados, no domicílio, utilizando-se de instrumento estruturado, baseado no questionário Older Americans Resources and Services (OARS), elaborado pela
Duke University e adaptado à realidade brasileira por Ramos ${ }^{(13)}$.

Antes de iniciar a entrevista, foi realizada avaliação cognitiva com o idoso, procurando verificar a preservação da memória recente para responder as questões propostas. Quando o idoso obteve menos de 13 pontos, solicitou-se um informante. Considerou-se como informante uma pessoa que reside na mesma casa que o entrevistado, e que soubesse oferecer informações sobre o idoso (entrevistado). Ao informante foi aplicada a escala PFEFFER. Quando o resultado foi cinco ou menos, a entrevista continuou sendo realizada com o idoso, e as informações complementadas pelo informante, denominado auxiliar. Quando o resultado foi seis ou mais, a entrevista foi conduzida com o informante, denominado substituto.

Todos os itinerários do entrevistador, no bairro, foram registrados na planilha de campo, e acompanhados por seu respectivo supervisor. Quando alguma informação mostrou-se duvidosa ou 0 processo de seleção dos domicílios tivesse tido algum problema, o supervisor orientava o entrevistador para que voltasse ao bairro, na referida residência, e procedessem as correções.

Nesta pesquisa, foram constituídos três grupos de idosos, de acordo com o local de residência nos Distritos Sanitários I, II e III. Para tal definição, utilizou-se um mapa do município de Uberaba disponibilizado pela Secretaria Municipal de Saúde contendo a divisão territorial dos Distritos Sanitários. Seguindo essa delimitação associou o bairro pertencente a cada distrito. No caso de bairros pertencentes a dois distritos sanitários, utilizouse um segundo critério: associar a rua ao distrito pertencente. Necessitou-se de um terceiro critério, pois havia ruas pertencentes a dois distritos sanitários. Assim, utilizou-se do endereço do idoso para identificação do distrito sanitário, baseando-se na Lista Telefônica GUIASEI - SABE.

As variáveis estudadas foram: demográficas e socioeconômicas (sexo, faixa etária, estado conjugal, escolaridade); saúde (auto-percepção da saúde, agravos à saúde no período de 12 meses, uso regular de 
Tavares DMS, Guidetti GECB, Saúde MIBM. Características sócio-demográficas, condições de saúde e utilização de serviços de saúde por idosos. Revista Eletrônica de Enfermagem [Internet]. 2008;10(2):299-309. Available from: http://www.fen.ufg.br/revista/v10/n2/v10n2a02.htm

medicamentos, problema para obter medicamento); uso de serviço de saúde (local de atendimento à saúde, consultas, internação, grau de satisfação com a internação e problemas no atendimento).

Conforme as entrevistas foram sendo realizadas, os instrumentos preenchidos foram entregues aos pesquisadores, que procederam as devidas revisões e codificações. Sempre que necessário, as fichas de entrevistas foram devolvidas para o entrevistador complementar as informações.

Foi construída uma base de dados, no programa Epi Info 3.2TM, e os dados coletados foram digitados em microcomputador, por duas pessoas, em dupla entrada, para posterior verificação da existência de registros de idosos duplicados, assim como de nomes diferentes entre as duas bases de dados. Procedeu-se então, à consistência dos campos verificando digitação errada. Quando havia dados inconsistentes retornava-se para a entrevista original, para confirmação e/ou correção.

Para atender aos objetivos propostos, foi utilizada, inicialmente, análise descritiva a partir de freqüências simples absolutas e percentuais, medidas descritivas (média, mediana, desvio padrão e valores máximo e mínimo), de acordo com a normalidade dos dados e variáveis categóricas e numéricas. As associações entre variáveis categóricas foram estudadas a partir de testes não paramétricos como qui-quadrado e teste exato de Fisher. O nível de significância para todos os testes foi de $5 \%$.

Esta pesquisa foi aprovada pelo Comitê de Ética em Pesquisa com Seres Humanos da Universidade Federal do Triângulo Mineiro, protocolo n0553, atendendo à Resolução 196/96 do Conselho Nacional de Saúde. Os idosos foram contactados em seus domicílios, aos quais foram apresentados os objetivos, o termo de consentimento livre e esclarecido, e oferecidas as informações pertinentes. Somente após a anuência do entrevistado e assinatura do termo de consentimento livre e esclarecido, foi conduzida a entrevista.

\section{RESULTADOS E DISCUSSÃO}

Os dados serão apresentados e discutidos, na seqüência dos objetivos propostos, apresentando a descrição dos idosos e a comparação entre os distritos sanitários.

Do total das entrevistas realizadas, 92\% foram com idosos, 4,2\% com informante auxiliar (houve contribuição nas respostas de um membro familiar ou cuidador que convive frequentemente com o idoso) e 3,8\% com informante substituto (na impossibilidade da entrevista ser realizada com o idoso, essa foi obtida junto a um membro familiar ou cuidador que convive frequentemente com o idoso). As proporções de entrevistados, considerando-se as categorias "idosos", "informante auxiliar" e 'informante substituto", não apresentam diferenças significativas quando comparados por sexos nas faixas etárias : $60 \mid-70\left({ }^{2}=0,73\right.$; $\mathrm{p}=0,70) ; 70 \mid-80\left(\mathrm{x}^{2}=1,72 ; \mathrm{p}=0,42\right)$ e 80 e mais $\left(x^{2}=1,55 ; p=0,46\right)$. Entretanto, quando se analisa cada sexo separadamente, verifica-se que, conforme aumenta a faixa etária, ocorre aumento na proporção de entrevistadores "informante substituto", tanto para o sexo masculino $\left(x^{2}=64,75 ; \quad p<0,0001\right)$ quanto feminino $\left(x^{2}=173,7 ; p<0,0001\right)$. Destaca-se que, no sexo feminino, ocorre um aumento acentuado na proporção de "entrevistadores substitutos" entre as mulheres com 80 anos e mais de idades.

\section{Dados demográficos e socioeconômicos}

O percentual de idosos distribuídos nos três distritos sanitários correspondeu a 33,5\% para o DSI; $35,7 \%$ para o DSII e $30,8 \%$ para o DSIII. Do total, $36 \%$ são do sexo masculino e $64 \%$ do feminino. Apesar do município de Uberaba possuir maior percentual de idosas $(56,7 \%)$, como ocorre na maioria do país, é possível que $o$ fato da pesquisa ter sido realizada no domicílio tenha favorecido a obtenção de um percentual ligeiramente maior para as mulheres.

Contudo, não se pode negar a feminilização no envelhecimento, que vem se ampliando nas faixas etárias mais velhas ${ }^{(3)}$. Este fato deverá impulsionar ações em saúde e sociais direcionadas às necessidades destas mulheres, uma vez que elas ainda possuem menor escolaridade e renda, podendo comprometer sua sobrevivência com qualidade e sua integração social. 
Tavares DMS, Guidetti GECB, Saúde MIBM. Características sócio-demográficas, condições de saúde e utilização de serviços de saúde por idosos. Revista Eletrônica de Enfermagem [Internet]. 2008;10(2):299-309. Available from: http://www.fen.ufg.br/revista/v10/n2/v10n2a02.htm

Neste estudo, a distribuição da faixa etária foi de $52,7 \%$ para $601-70$ anos; $33,6 \%$ para $70 \mid-80$ e $13,7 \%$ para os idosos com 80 anos e mais. Os dados do IBGE (4) apresentam percentuais semelhantes para o município, em cada uma destas faixas etárias, correspondendo a $57,4 \%, 30,1 \%$ e $12,6 \%$, respectivamente. No Brasil, em dez anos houve crescimento, especialmente, dos idosos com 75 anos e mais, podendo este fato estar relacionado com os avanços na área da saúde ${ }^{(3)}$. Quando se comparam os dados de Uberaba por faixa etária com os do Brasil, após o referido crescimento, observam-se percentuais semelhantes, denotando que o município está vivenciando o fenômeno da longevidade. Ressalta-se que o país, os municípios, a sociedade e as famílias devem atentar para este fato e somar esforços para o enfrentamento desta questão. Sabe-se que os idosos são mais vulneráveis a presença de poli morbidades, que podem levar às incapacidades e dependências, e necessitam de mais atendimento à saúde. Ademais, os cuidados requerem medidas de bem-estar, disponibilidade de tempo e recursos financeiros dos familiares ${ }^{(3)}$.

A comparação entre os distritos sanitários evidenciou que os idosos pertencentes à faixa etária de 601- 70anos estão em maior proporção localizados no DSI enquanto nos DSII e DSIII há uma maior proporção de idosos com

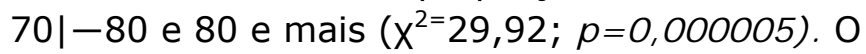
envelhecimento é definido como "processo seqüencial, individual, acumulativo, irreversível, universal, não patológico, de deterioração de um organismo maduro, próprio a todos os membros de uma espécie, de maneira que o tempo o torne menos capaz de fazer frente ao estresse do meio-ambiente e, portanto, aumente sua possibilidade de morte" ${ }^{\prime(14)}$. Baseando-se nesta definição, há maior possibilidade do idoso apresentar declínio funcional e doenças conforme aumenta a sua idade. Assim, as ações em saúde direcionadas aos idosos do DSII e III devem estar voltadas para a identificação precoce da fragilidade em idosos com idade mais avançada. Ademais, o declínio da saúde pode gerar isolamento social e solidão, agravando ainda mais a qualidade de vida do idoso(1). Quanto à menor longevidade dos idosos residentes no Distrito Sanitário I, pode estar associada à maior precariedade deste território no que se refere a indicadores econômicos e sócio-sanitários.

Quanto ao estado conjugal, os maiores percentuais foram para os idosos que moram com esposo ou companheiros (47\%) e viúvos $(36,6 \%)$, o que indica uma maior presença do apoio familiar. Sabe-se que a permanência dos idosos em seus núcleos familiares e comunitários contribui para o seu bem-estar ${ }^{(2)}$. A equipe multidisciplinar deve aprofundar sobre este aspecto para que possa identificar o cuidador e reforçar o apoio familiar.

A escolaridade apresentou mediana de três anos, com valores mínimos de zero e máximo de vinte e três. Categorizando por faixa, verificou-se que os maiores percentuais são para aqueles que possuem 4 a 8 anos $(35,3 \%)$, seguidos pelos que possuem 1 a 3 anos $(30,5 \%)$ e sem escolaridade $(23,08 \%)$. A escolaridade tem sido associada à melhoria na qualidade de vida e longevidade. Verificou-se no Brasil um aumento percentual de 10 pontos na taxa de analfabetismo nos últimos dez anos ${ }^{(3)}$. Neste estudo, o percentual obtido para os idosos sem escolaridade está abaixo dos encontrados para o Estado de Minas Gerais $(34,3 \%)$ e para o Brasil $(33,5 \%)^{(3)}$.

Existe uma maior proporção de idosos sem escolaridade no DSI, enquanto os DSII e DSIII possuem maior proporção de idosos com nove anos ou mais de estudo $\left(X^{2}=88,42 ; p=\right.$ $0,000000)$. A escolaridade influencia no cuidado à saúde, pois, o analfabetismo está associado ao risco de deficiência e morte ${ }^{(1)}$. Assim, os serviços de saúde localizados no DSI, devem desenvolver estratégias para lidar com esta questão, de forma a incentivar os idosos ao autocuidado e envolver os familiares na atenção ao idoso.

\section{Condições de saúde}

A autopercepção da saúde atual foi considerada, pela maioria, como regular $(43,5 \%)$ e boa $(39,9 \%)$. Observou-se que grande parte dos idosos considerou sua saúde, quando comparada com 12 meses atrás, inalterada $(59,4 \%)$, entretanto, 24,4\% considerou-a pior e $15,6 \%$ melhor. Estudo 
Tavares DMS, Guidetti GECB, Saúde MIBM. Características sócio-demográficas, condições de saúde e utilização de serviços de saúde por idosos. Revista Eletrônica de Enfermagem [Internet]. 2008;10(2):299-309. Available from: http://www.fen.ufg.br/revista/v10/n2/v10n2a02.htm

realizado pelo IBGE (17) verificou que a autopercepção de saúde considerada muito boa e boa diminuiu de acordo com o aumento da idade, chegando a percentuais, entre os idosos com idade acima de 64 anos, de $42,3 \%$, para homens, e $39,6 \%$, para as mulheres. Verificouse também que ocorrem variações de acordo com o rendimento, apresentando percentuais maiores para os que possuem maiores salários. No contexto da saúde, a autopercepção considerada ruim aumenta o risco de mortalidade, interfere na satisfação com a vida e no bem-estar subjetivo ${ }^{(16)}$.

No DSIII os idosos apresentaram a autopercepção atual da saúde classificada em boa e ótima, enquanto que nos outros dois distritos, consideram-na boa e regular $\left(x^{2}=\right.$ $68,57 ; p=0,000000)$. Entretanto, quando a compara com 12 meses atrás, a maior proporção de idosos do DSI qualifica a situação atual como pior $\left(X^{2}=76,84 ; p=0,000000\right)$. Dada a influência da autopercepção da saúde na vida do idoso referida acima, seria necessário o desenvolvimento de outra pesquisa para compreender os fatores que têm contribuído para a piora desta autopercepção entre aqueles residentes no DSI, de forma a implementar ações efetivas, visando minimizar tal situação.

Obteve-se que $77,7 \%$ dos idosos utilizam medicamentos regularmente obedecendo a receitas medicas, entretanto $1,98 \%$ dos idosos fazem uso regular de medicamentos sem prescrição médica. Os problemas para a aquisição de medicamentos foram referidos por $77,6 \%$ dos idosos que utilizam remédios regularmente, destacando-se o custo $(58,7 \%)$. Apesar da Lei no 9.787, de 1999 (Lei dos Medicamentos Genéricos) ${ }^{(17)}$ estimular a concorrência e a variedade de oferta no mercado de remédios, a melhoria da qualidade dos medicamentos, a redução nos preços e facilitar o acesso da população aos tratamentos, percebe-se que a população idosa ainda encontra dificuldades para adquirir estes produtos. Considerando as polimorbidades, por vezes presente nos idosos, e os baixos rendimentos, cabe ao profissional de saúde contribuir na divulgação, prescrição e somar esforços, com a população idosa, para implementação efetiva da legislação dos medicamentos genéricos.

Observou-se que o DSIII possui maior proporção de idosos sem problemas na obtenção de medicamentos comparada aos do DSI e DSII $\left(X^{2}=28,19 ; p=0,000001\right)$. É provável que este fator esteja sendo influenciado pela renda mensal do idoso, uma vez que os medicamentos oferecidos pelos serviços de saúde foram distribuídos, durante período do estudo, com descontinuidade. No município de Uberaba, $51,7 \%$ dos idosos possui renda individual de um salário mínimo, seguidos pelos que possuem 1 a 3 salários mínimo $(21,5 \%)$ e sem renda $(12,5 \%)^{(18)}$.

\section{Uso de serviços de saúde}

A maioria dos idosos (62,3\%) depende dos serviços públicos, estaduais ou municipais, de saúde para seu atendimento, seguidos pelo convênio particular (19,1\%), Hospital Escola da UFTM $(10,5 \%)$ e médico particular $(3,8 \%)$. Verificou-se que no DSI há maior proporção de idosos usuários dos serviços municipais/ estaduais comparado aos outros dois distritos $\left(X^{2}=57,52 ; p=0,0000000\right)$. Ressalta-se que o Hospital Escola é um serviço de saúde do SUS, porém nesse estudo foi colocado como opção separada, por estar ligado à Universidade em que foi realizada esta pesquisa, e não ser porta de entrada, já que é um serviço de referência. Portanto, observa-se que, aproximadamente, $70 \%$ dos idosos dependem do SUS para realizarem a atenção à sua saúde. O mesmo ocorre em todo território nacional, como visualizado na pesquisa realizada com todas as faixas etárias. Os principais locais de atenção à saúde foram unidades de saúde $(52,4 \%)$, consultórios particulares $(18 \%)$, ambulatórios de hospitais $(16,9 \%)$, pronto socorro ou emergência $(5,8 \%)$, ambulatório ou consultório de clínicas $(4,4 \%)$ e farmácia $(1,4 \%)^{(15)}$.

A procura no serviço de saúde no último ano foi referida por $70,4 \%$ dos idosos, dos quais $66,5 \%$ são mulheres e $33,5 \%$ homens. Estudos demonstram que os homens procuram menos os serviços de saúde para as ações de promoção da saúde e preventivas de doença, pois podem associar a fraqueza ao comportamento feminino, levando às 
Tavares DMS, Guidetti GECB, Saúde MIBM. Características sócio-demográficas, condições de saúde e utilização de serviços de saúde por idosos. Revista Eletrônica de Enfermagem [Internet]. 2008;10(2):299-309. Available from: http://www.fen.ufg.br/revista/v10/n2/v10n2a02.htm

desconfianças da masculinidade, socialmente instituída. Outras questões seriam o medo da descoberta de uma doença grave e a vergonha de expor o seu corpo ${ }^{(19)}$.

Para a análise dos problemas de saúde que motivaram a procura pelo serviço de saúde no último ano, considerou-se somente o primeiro problema citado por cada idoso.

Nos últimos doze meses, $70 \%$ dos idosos tiveram problemas de saúde e procuraram os serviços, e os principais motivos foram consultas médicas $(68,7 \%)$, urgências/emergências $(13,6 \%)$, exames de rotinas $(9 \%)$, procedimentos de enfermagem $(3,8 \%)$ e outros $(4,9 \%)$. $\mathrm{Na}$ população brasileira a procura pelo serviço de saúde representou $62 \%$. Verificou-se que o número de consultas médicas realizadas no ano aumentava proporcionalmente à idade ${ }^{(15)}$.

No DSI, os idosos procuram, em maior proporção, os serviços para consultas médicas e de urgência e emergência, enquanto que nos DSII e DSIII a procura decorre para exames de rotina $\left(x^{2}=15,87 ; p=0,003199\right)$. No DSI devem-se investigar as causas da procura pelos serviços de urgência e emergência, visando identificar se decorrem de possíveis fatores de risco presentes no território ou se são provenientes da falta de resolutividade das unidades de atenção básica. Ademais, devemse implementar estratégias no DSI que propiciem a conscientização pela busca de serviços promocionais de saúde e preventivos de doenças.

A satisfação com o atendimento à saúde foi referida por $76 \%$ dos idosos. Dentre aqueles que estiveram internados no último ano $(21,5 \%), 73,1 \%$ referiram que ficaram satisfeitos e muito satisfeitos. Ressalta-se que $67 \%$ das pessoas internadas eram mulheres. No Brasil, o maior coeficiente de internação ocorreu no grupo etário com 65 anos ou mais $(14,0$ por 100 pessoas no grupo), seguido pelo grupo etário de 0 a 4 anos $(9,1$ por 100 pessoas no grupo). Os coeficientes de internação hospitalar apresentaram decréscimo entre 0 grupo de menor rendimento familiar $(8,4$ por 100 pessoas no grupo) até o grupo de 3 a 5 salários mínimos $(6,3$ por 100 pessoas no grupo) e se estabilizaram a partir daí. Vale destacar que as pessoas sem rendimento foram as que apresentaram o maior coeficiente de internação hospitalar (10,6 por 100 pessoas no grupo $)^{(15)}$.

No DSI e no DSII há maior proporção dos idosos satisfeitos com 0 atendimento nos serviços de saúde, enquanto no DSIII estão muito insatisfeitos $\left(X^{2}=28,30 ; p=0,000420\right)$. 0 mesmo se observa em relação à satisfação com a internação $\left(X^{2}=18,90 ; p=0,004329\right)$. Cabe identificar no DSIII, quais são os fatores que tem contribuído para tal insatisfação.

Os problemas enfrentados nos serviços de saúde foram referidos por $56 \%$ dos idosos, estando principalmente relacionados ao longo tempo de espera (72\%); à dificuldade de ser atendido quando precisa $(12 \%)$; ao fato do atendimento não ser bom ( $5 \%)$, e por não ter confiança no atendimento (2\%). Na população brasileira, os motivos para a não procura dos serviços de saúde foram a falta de dinheiro $(23,8 \%)$, atendimento demorado $(18,1 \%)$, serviço distante ou o acesso/transporte difícil $(12,7 \%)$ e horário incompatível com o seu $(12,7 \%)^{(15)}$.

Os problemas com 0 atendimento nos serviços de saúde são diferenciados, de maneira que no DSI estão relacionados ao longo tempo de espera para ser atendido, no DSII é a distância dos serviços de saúde e no DSIII consideram difícil ser atendido quando precisa $\left(X^{2}=102,66 ; p=0,000001\right)$. A longa espera para ser atendido, referida pelos idosos no DSI, pode dever-se à procura preferencial por serviços de urgência e emergência, nos quais o tempo de espera é, usualmente, maior.

Estes dados evidenciam a necessidade de reorganizar a atenção à saúde da população idosa do município. De acordo com o Plano Municipal de Saúde ${ }^{(20)}$, estão previstas as estratégias de implantar protocolo de atenção à saúde do idoso; desenvolver ações de educação permanente voltadas para trabalhadores e gestores de saúde, focalizando o processo de envelhecimento populacional; criar Centro de referência municipal para atenção à saúde do idoso, em parceria com a Secretaria de Desenvolvimento Social até 2009 e propor parcerias, aos equipamentos sociais localizados nos bairros dos três distritos sanitários, para oferecer ao idoso espaço para práticas de 
Tavares DMS, Guidetti GECB, Saúde MIBM. Características sócio-demográficas, condições de saúde e utilização de serviços de saúde por idosos. Revista Eletrônica de Enfermagem [Internet]. 2008;10(2):299-309. Available from: http://www.fen.ufg.br/revista/v10/n2/v10n2a02.htm

promoção a saúde, como natação, hidroginástica, entre outras.

Em síntese, considerando o local de residência dos sujeitos que participaram deste estudo, podemos afirmar que o percentual de idosos nos três distritos sanitários é de 33,5\% para o DSI; $35,7 \%$ para o DSII e $30,8 \%$ para o DSIII. A maioria é do sexo feminino (64\%); distribuídos em $52,7 \%$ para faixa etária de $60 \mid-70$ anos; $33,6 \%$ para $70 \mid-80$ e $13,7 \%$ para os idosos com 80 anos e mais; $47 \%$ moram com esposo ou companheiros, e $36,6 \%$ são viúvos.

Os idosos residentes no DSI estão em maior proporção na faixa etária de $60 \mid-70$ anos; sem escolaridade; apresentam autopercepção atual da saúde classificada como boa e regular e quando comparada com 12 meses atrás, referem-na como pior; apresentam mais problemas na obtenção de medicamentos que os do DS II e III; freqüentam mais os serviços municipais/ estaduais; procuram os serviços para consultas médicas e urgência e emergência; estão mais satisfeitos com o atendimento e a internação, apresentam mais problemas no atendimento dos serviços de saúde, relacionados ao longo tempo de espera para ser atendido.

Os idosos residentes no DSII estão em maior proporção na faixa etária de $70 \mid-80$ e 80 e mais; com escolaridade de nove anos ou mais de estudo; apresentam autopercepção atual da saúde classificada como boa e regular; apresentam mais problemas na obtenção de medicamentos que os do DS III; procuram os serviços para exames de rotina; estão mais satisfeitos com o atendimento e a internação, apresentam menos problemas no atendimento dos serviços de saúde e quando os têm, estes se devem a distância dos serviços de saúde.

Os idosos residentes no DS III estão em maior proporção na faixa etária de $70 \mid-80$ e 80 e mais; com escolaridade de nove anos ou mais de estudo; apresentam autopercepção atual da saúde classificada como boa e ótima; apresentam menos problemas na obtenção de medicamentos; procuram os serviços para exames de rotina; estão insatisfeitos com o atendimento e a internação, apresentam menos problemas no atendimento dos serviços de saúde e, quando os têm, estes se devem ao fato de ser difícil o atendimento quando precisa.

\section{CONSI DERAÇÕES FI NAIS}

Verificou-se, neste estudo, diferenças nas variáveis demográficas, socioeconômicas e de saúde entre os idosos residentes no município. Estas diferenças já haviam sido identificadas por estudo realizado, na década de 90, pela secretaria de saúde, que balizaram a definição de áreas homogêneas do ponto de vista sóciosanitário em cada um dos Distritos Sanitários. Nesta época, o DSI era o que concentrava a maior extensão territorial de áreas precárias. Estes dados não estão atualizados e também não há informações desagregadas por distrito sanitário, o que enriqueceria a discussão deste trabalho.

O DSI, apesar de ter uma população idosa mais jovem, apresenta maiores desafios na atenção à sua saúde. Sabe-se que a baixa escolaridade e renda interferem no processo de adoecer, através da dificuldade de acesso ao serviço de saúde, das iatrogenias presentes nos serviços de saúde, no déficit do autocuidado, na dificuldade de adesão ao tratamento - tanto por questões financeiras e por resistência a valores e determinações impostos pela classe hegemônica, quanto à consciência sanitária. No entanto, estão mais satisfeitos com o atendimento $e$ as internações. Este fato possibilita alguns questionamentos, que necessitarão de estudos posteriores para serem esclarecidos. A satisfação dos idosos com o atendimento e internação no DSI estaria relacionada à maior resolutividade dos serviços de saúde presentes neste território? Por outro lado, os idosos do DSI buscam, prioritariamente, serviços de urgência e emergência. $O$ que estes serviços têm ofertado? Sendo o DSI o território com o maior número de unidades de atenção básica, por que seus idosos não demandam este atendimento?

Buscou-se, neste estudo, verificar em que os idosos residentes nos três distritos sanitários apresentam diferenças, visando subsidiar o planejamento das ações em saúde, atendendo às especificidades deste grupo populacional, gerar novas questões para futuras pesquisas e 
Tavares DMS, Guidetti GECB, Saúde MIBM. Características sócio-demográficas, condições de saúde e utilização de serviços de saúde por idosos. Revista Eletrônica de Enfermagem [Internet]. 2008;10(2):299-309. Available from: http://www.fen.ufg.br/revista/v10/n2/v10n2a02.htm

possibilitar a discussão e reflexão sobre a rede de serviços de saúde do município.

\section{REFERÊNCI AS}

1. World Health Organization. Envelhecimento ativo: uma política de saúde. World Health Organization; Brasília: Organização PanAmericana da Saúde, 2005.

2. Ministério da Saúde. Estatuto do Idoso. Brasília: Ministério da Saúde, 2006.

3. Instituto Brasileiro de Geografia e Estatística. Estudos e Pesquisas Informação Demográfica e Socioeconômica número 21. Síntese de Indicadores Sociais. Uma Análise das Condições de Vida da População Brasileira. Rio de Janeiro: IBGE, 2007.

4. Instituto Brasileiro de Geografia e Estatística [Internet]. Brasília: Ministério do Planejamento, Orçamento e Gestão (BR) [cited 2007 out 12]. Pesquisa Nacional por Amostra de Domicilio. Síntese de indicadores 2006. Available from: http://www.ibge.gov.br/home/estatistica/populacao/t rabalhoerendimento/pnad2006/sintesepnad2006.pdf. 5. Tavares DMS, Pereira GA, Iwamoto $\mathrm{HH}$, Miranzi SCC, Rodrigues LR, Machado ARM. Incapacidade funcional entre idosos residentes em um município do interior de Minas Gerais. Texto Contexto Enferm. 2007;16(1):32-9.

6. Ministério da Saúde (BR). Portaria no. 2.528. Aprova a Política Nacional de Saúde da Pessoa Idosa. Diário Oficial da União. 2006 out 20.

7. Motta LB, Aguiar AC. Novas competências profissionais em saúde e o envelhecimento populacional brasileiro: integralidade, interdisciplinaridade e intersetorialidade. Rev Ciência \& Saúde Coletiva. 2007;12(2):363-372.

8. Silvestre JAS, Costa Neto MMC. Abordagem do idoso em programas de saúde da família. Cad. Saúde Publica. 2003;19(3):839-47.

9. Rezende DA. Planejamento de informações públicas municipais: sistema de informação e de conhecimento, informática e governo eletrônico integrados aos planejamentos das prefeituras e municípios. Rev. Adm. Pública. 2007;41(3):50536.

10. Pessoto UC, Heimann LS, Boaretto RC, Castro IEN, Kayano J, César IL, et al. Desigualdades no acesso e utilização dos serviços de saúde na região metropolitana de
São Paulo. Rev Ciência \& Saúde Coletiva. 2007;12(2):351-362.

11. Prefeitura Municipal de Uberaba [Internet]. Uberaba: Secretaria de Dezenvolvimento Econômico e Turismo (BR) [cited 2007 ago 08]. Uberaba em dados. Edição 2007. Ano Base 2006. Available from: http://www.uberaba.mg.gov.br/sedet/uberaba em dados 2007/Uberaba.em.Dados.2007cap.03-Indicadores Sociais.pdf.

12. Instituto Brasileiro de Geografia e Estatística [Internet]. Brasília: Ministério do Planejamento, Orçamento e Gestão (BR) [cited 2007 out 10]. Cidades@. Available from: http://www.ibge.gov.br/cidadesat/default.php

13. Ramos LR. Growing old in São Paulo, Brazil. Assessment of Health status and family support of the elderly of different socio-economic strata living in the community [thesis]. London (England): London School of Hygiene and Tropical Medicine; 1987.

14. Organização Panamericana de la Salud. Guia clínico para atención primaria a las personas mayores. 3 ed. Washington: OPAS, 2003.

15. Instituto Brasileiro de Geografia e Estatística [Internet]. Brasília: Ministério do Planejamento, Orçamento e Gestão (BR) [cited 2007 out 12]. Pesquisa nacional por amostra de domicílios. Acesso e Utilização de Serviços de Saúde 2003. Available

from: http://www.ibge.gov.br/home/estatistica/populacao/t rabalhoerendimento/pnad2003/saude/saude2003.pdf 16. Kikuchi, El. Auto-avaliação da saúde. In: Jacob Filho, W; Amaral, JRG. Avaliação global do idoso: manual da liga de Gamia. São Paulo: Atheneu; 2005. p. 25-31.

17. Presidência da Republica (BR). Lei no. 9.787. Dispõe sobre a regulamentação dos Medicamentos Genéricos. Diário Oficial da União 1999 fev 10.

18. Tavares DMS, Pereira GA, Iwamoto $H H$, Miranzi SCC, Rodrigues LR, Machado ARM. Relatório final da pesquisa: condições de saúde de idosos residentes no município de Uberaba. Uberaba: 2006.

19. Gomes R, Nascimento EF, Araújo FC. Por que os homens buscam menos os serviços de saúde do que as mulheres? As explicações de homens com baixa escolaridade e homens com 
Tavares DMS, Guidetti GECB, Saúde MIBM. Características sócio-demográficas, condições de saúde e utilização de serviços de saúde por idosos. Revista Eletrônica de Enfermagem [Internet]. 2008;10(2):299-309. Available from: http://www.fen.ufg.br/revista/v10/n2/v10n2a02.htm

ensino superior. Rev Cad. Saúde Pública. 2007;23(3):565-74.

20. Secretária municipal de saúde. Plano

Municipal de Saúde de Uberaba. Uberaba: 2007.

Artigo recebido em 01.02 .08

Aprovado para publicação em 30.06.08 